\title{
关于 Amann 的一个定理的注记
}

设 $E$ 是 Banach 空间, $P$ 为 $E$ 中雉,

$$
f: R \times P \rightarrow P \text {. }
$$

关于算子方程 $x=f(\lambda, x)$ 多重解的存在性, H. Amann 进行了深入的研究, 他证明了

定理 (H. Amann) 设 $E$ 为 Banach 空间, $P$ 为 $E$ 中正规体锥, $f: R^{+} \times P \rightarrow P$ 全连续, 二次连续右 可微, $f(0,0)=0, \lambda^{*}=\sup \left\{\lambda \in R^{+}: \exists x \in P\right.$ 使

$$
x=f(\lambda, x)\}<+\infty \text {. }
$$

且 $\forall(\lambda, x) \in\left(0, \lambda^{*}\right) \times \rho$ 时, $f^{\prime}(\lambda, x)=\left(f_{2}(\lambda, x), f_{x}(\lambda\right.$, $x)): R \times E \rightarrow E$ 强正. 若 $\exists \mu \in\left(0, \lambda^{*}\right), \rho>0$ 使

$$
\lambda \in\left[\mu, \lambda^{*}\right]
$$

且 $x \in P,\|x\| \geqslant \rho$ 时, $x \neq f(\lambda, x)$. 那么

$$
\forall \lambda \in\left[\mu, \lambda^{*}\right), x=f(\lambda, x)
$$

在 $P$ 中至少有两个解.

本文利用新的方法研究 $x=f(\lambda, x)$ 多重解的 存在性,大大简化了上述定理的证明,减弱了定理的 条件，指出关于 $t$ 的可微性假设是不必要的(这个条 件使得不能在 $f$ 凝聚的情况讨论多重解的存在性). 详言之,本文证明了

定理设 $E$ 为 Banach 空间, $P$ 为 $E$ 中正规体
锥, $t: R^{+} \times P \rightarrow P$ 全连续, $f(0,0)=0, \forall \lambda \in R^{+}$, $f(\lambda, \cdot): P \rightarrow P$, 增 $\forall x \in P, f(\cdot, x): R^{+} \rightarrow P$ 强增. 如 $\lambda^{*}<+\infty, \exists \mu \in\left(0, \lambda^{*}\right), \rho>0$, 使 $\lambda \in\left[\mu, \lambda^{*}\right]$, $x \in P$ 且 $\|x\| \geqslant \rho$ 时, $x \neq f(\lambda, x)$. 那么 $\forall \lambda \in\left[\mu, \lambda^{*}\right)$, $x=f(\mu, x)$ 在 $P$ 中至少有两个解.

本文方法的关键是利用下述引理.

引理设 $E$ 为 Banach 空间, $P$ 为 $E$ 中正规 体锥, $f: R^{+} \times P \rightarrow P$ 全连续, 增, $\left(\lambda_{0}, x_{0}\right) \in R^{+} \times P$, $x_{0}=f\left(\lambda_{0}, x_{0}\right), x_{1} \gg x_{0}, f\left(\lambda_{0}, x_{1}\right) \leqslant x_{1}$. 若 $\exists R>0$. 使 $r \geqslant R$ 时, $i\left(h, r B_{+}\right)=0$, 其中

$$
h(x)=f\left(\lambda_{0}, x_{0}+x\right)-x_{0},
$$

$r B_{+}=\{x \in P:\|x\|<r\}$. 那么, $\exists x^{*}>x_{0}$, 使

$$
f\left(\lambda_{0}, x^{*}\right)=x^{*} \text {. }
$$

值得指出的是,由于我们不要求 $t$ 有可微性, 用 我们的方法可将上述结果推广到 $f$ 凝聚的情况. 因 此, 我们的方法提供了一种研究凝聚算子方程多重 解的途径.

\section{具有小时滞的非线性微分差分方程解的稳定性}

早在五十年代, 就有不少作者对微分差分方程 的解与常微分方程的解在稳定性方面的关系进行了 探讨. 但只是对线性自治的微分差分方程得出了较 为理想的结果, 对于一般的微分方差分程至今没有 这方面的结果. 1955 年, E. M. Wright 讨论了最 简单的微分差分方程:

$$
\dot{x}(t)=-\alpha x(t-r), \alpha>0 r>0 x \in R^{\prime},
$$

其结果为: 若 $\alpha r<\pi / 2$, 则方程的平凡解一致渐近 稳定. 1958 年, 秦元勋讨论了方程

$\dot{x}(z)=a x(t)+b x(t-r) a, b$ 是实常数, $r>0$, 得出若 $a+b<0,0<r<\pi / 8(|a|+|b|)$, 则方程 的平凡解一致渐近稳定. 1959 年, 秦元勋等人对 $n$ 阶方程组

$$
\dot{x}_{i}(t)=\sum_{i=1}^{n} a_{i j} x_{j}(t)+\sum_{i=1}^{n} b_{i j} x_{j}\left(t-r_{i i}\right),
$$

$$
i=1,2, \cdots n \text {. }
$$

进行了讨论, 其中 $a_{i}, b_{i j}, r_{i j}$ 是实常数, $r \geqslant r_{i}>0$, 当 $r>0$ 充分小时,方程组的平凡解的渐近稳定性可 由对应的常微分方程组的平凡解的渐近稳定性推 出. 其证明方法都是用特征方程的恨的分布状况和. 复变函数的一些理论.

本文讨论一般的非线性微分差分方程

$$
\dot{x}(t)=f(t, x(t), x(t-r)), r>0,
$$
其中 $f: R^{i} \times R^{n} \times R^{x} \rightarrow R^{n}$. 通过对应的常微分方程

$$
\dot{x}(t)=f(t, x(t), x(t))
$$

的解的性质来讨论方程 (1) 的解的性质.

众所周知, 在对 $f$ 的某些假设下,若方程 (2) 的 平凡解是一致渐近稳定的 (一切解一致有界且一致 毕竟有界), 则存在 Ляпунов 函数 $V(t, x)$, 满足某 些性质. 我们将这样的 ЈІяпунов 函数用于方程 
(1)，然后应用关于泛函微分方程的 Разумихин 型 定理,我们得到如下结果:

定理 1 设 $f: R^{1} \times R^{r} \times R^{n} \rightarrow R^{n}$ 连续, 关于第 二个变量满足 Lipschitz 条件, 关于第三个变量具有 一致有界的连续偏导数, $f(t, 0,0)=0\left(t \in R^{1}\right)$, 若 方程 (2) 的平凡解是全局指数渐近稳定的, 则存在 $r_{0}>0$, 使 $0<r \leqslant r_{0}$ 时, 方程 (1) 的平风解是全局一
致渐近稳定的。

定理 2 设 $f: R^{1} \times R^{n} \times R^{n} \rightarrow R^{n}$ 满足定理 1 的假设 (除 $f(t, 0,0)=0$ 外), 若方程 (2) 的解是一致 有界和一致毕竟有界的, 则存在 $r_{0}>0$, 使 $0<r \leqslant r_{0}$ 时,方程 (1) 的解也是一致有界和一致毕竟有界的.

胡作生

（山东大学数学系,济南）

\section{随机线性方程组的一个极限性质*}

考虑随机线性方程组

$$
X_{n}=V_{n}+\frac{1}{n} W_{n} X_{n},
$$

这儿 $w_{n}=\left(w_{i j}\right)_{r \times n}, w w_{i j}, i, j=1,2, \cdots$ 为一列 iid 随机变量序列且 $E w_{i j}=0 . \quad V_{n}=\left(\alpha_{1}, \cdots, \alpha_{n}\right)^{\prime}$ 为 $n \times 1$ 列向量, $\left\{\alpha_{n}\right\}, n=1,2, \cdots$ 为一列常数序 列. 这类方程组在一些物理大系统中起着十分重 要的作用. Gernan 和 Hwang (参见 Z. Wahrsch. Verw. Gekiete, 60 (1981)，291-314）首先在 $w_{11}$ 的八阶矩存在的条件下证明了解的存在性及解的极 限性质，白志东随后在二阶矩存在的条件下证得了 比 Geman 和 Hwang 更强的结论. 最近, 作者用截 尾法进一步研究了这一问题, 把条件又大大减弱到 几乎不能再减弱的地步. 具体地我们有:

定理 1 若 $I-\frac{W_{n}}{n}$ 非奇异, 则解 $X_{n}$ 由(1.1) 式确定, 若 $l-\frac{W_{n}}{n}$ 奇异, 则定义 $X_{n}=0$. 设

$$
E w_{11}=0, E\left|w_{11}\right|^{1+r}<\infty,
$$

这儿 $0<r \leqslant 1$, 以及 $\sum_{i=1}^{\infty} \alpha_{i}^{2}<\infty$, 则

$$
\sum_{i=1}^{n}\left(x_{n i}-\alpha_{i}\right)^{2} \rightarrow 0 \text {, a.s. }(n \rightarrow \infty) \text {. }
$$

定理 2 设 $r \varepsilon\left(\frac{1}{2}, 1\right)$,

$$
E w_{11}=0, E w_{11}^{2} \triangleq \sigma^{2}<\infty \text {, }
$$

以及 $\sum_{i=1}^{n} \alpha_{i}^{2}<\infty$, 当 $I_{n}-\frac{w_{n}}{n^{r}}$ 非奇异时, $X_{n}$ 由如 下随机线性方程组确定

$$
X_{n}=V_{n}+\frac{1}{n^{r}} W_{n} X_{n},
$$

否则令 $X_{n}=0$, 则解 $X_{n}$ 满足

$$
\sum_{i=1}^{n}\left(x_{n i}-\alpha_{i}\right)^{2} \rightarrow 0 \text {, a.s. }(n \rightarrow \infty) \text {. }
$$

以上 $X_{n}=\left(x_{n 1}, x_{n 2}, \cdots, x_{n n}\right)^{\prime}$ 为 $n \times 1$ 列向 量.

缪柏其

(中国科学技术大学教学系,合肥)

*中国科学院科学基金资助的课题.

\section{关于黎曼空间的一类共形映照}

对于二黎曼空间 $(M, g)$ 和 $(\bar{M}, \bar{g})$, 若它们的 度量张量满足

$$
\left.\overline{\mathrm{g}}_{i j}=\rho^{2} g_{i j} \quad 1 \leqslant i, j, k, l \leqslant n\right),
$$

其中 $\rho$ 是 $x$ 的任一纯量函数, 则我们称 $M$ 和 $\bar{M}$ 之间 的这样的对应为共形映照. 如所知, 共形映照 (1) 为 保圆映照的充要条件是函数 $\rho$ 满足微分方程

$$
\rho_{i j}=\phi g_{i j} \text {, }
$$

第 8 期
这里

$$
\stackrel{\text { 这里 }}{\rho_{i j}}=\nabla_{i} \rho_{i}-\rho_{i} \rho_{j}+\frac{1}{2} g^{k l} \rho_{k} \rho_{l} g_{i j}, \rho_{i}=\frac{\partial \ln \rho}{\partial x^{i}} .
$$

在这一情形下, 张量 $\rho_{i j}$ 在 $M$ 的主方向是不定的. 同 时, 这个映照将每个测地圆变到测地圆.K. Yano 还 得到了保圆曲率张量

$$
Z_{j k l}^{i} \equiv R_{j k l}^{i}-\frac{R}{n(n-1)}\left(g_{j k} \delta_{i}^{i}-g_{i l} \delta_{k}^{i}\right) .
$$

科学 通报 